STUDI

FRANCESI

\section{Studi Francesi}

Rivista quadrimestrale fondata da Franco Simone

166 ( | LVI) | 2012

Varia

\title{
Comment peut-on être sceptique? Hommage à Didier Deleule. Études réunies par Michèle Cohen-Halimi et Hélène L'Heuillet
}

\section{Peter Balazs}

\section{(2) OpenEdition \\ Journals}

Édition électronique

URL : https://journals.openedition.org/studifrancesi/4678

DOI : $10.4000 /$ studifrancesi.4678

ISSN : 2421-5856

Éditeur

Rosenberg \& Sellier

\section{Édition imprimée}

Date de publication : 1 avril 2012

Pagination : 145-146

ISSN : 0039-2944

\section{Référence électronique}

Peter Balazs, «Comment peut-on être sceptique? Hommage à Didier Deleule. Études réunies par Michèle Cohen-Halimi et Hélène L'Heuillet », Studi Francesi [En ligne], 166 (I | LVI) | 2012, mis en ligne le 30 novembre 2015, consulté le 19 novembre 2021. URL : http://journals.openedition.org/studifrancesi/ 4678 ; DOI : https://doi.org/10.4000/studifrancesi.4678

Ce document a été généré automatiquement le 19 novembre 2021.

\section{cc) (†) $\odot$}

Studi Francesi è distribuita con Licenza Creative Commons Attribuzione - Non commerciale - Non opere derivate 4.0 Internazionale. 


\title{
Comment peut-on être sceptique? Hommage à Didier Deleule. Études réunies par Michèle Cohen-Halimi et Hélène L'Heuillet
}

\author{
Peter Balazs
}

\section{RÉFÉRENCE}

Comment peut-on être sceptique? Hommage à Didier Deleule. Études réunies par Michèle COHEN-HALIMI et Hélène L'HEUILLET, Paris, Honoré Champion, 2010, pp. 320.

Le volume, qui réunit les études des anciens élèves de Didier Deleule, tourne autour de la question du scepticisme et des conditions de la possibilité de la recherche philosophique. Un article inédit de Didier DELeULE, intitulé Scepticisme et politique (pp. 7-22) ouvre le volume. Dans cette étude - dans laquelle la gamme des penseurs cités s'étend de La Mothe le Vayer jusqu'à Oakeshott - le philosophe aborde la question épineuse des manifestations historiques et seulement possibles d'une attitude sceptique dans le domaine de la politique. L'étude de Frédéric BRAHAMI ("Seule la nature sait" - sur le Hume de Deleule, pp. 25-36) reprend, avec un recul de trente ans, le grand livre de Deleule sur Hume et la naissance du libéralisme économique. Brahami voit le mérite principal de cet ouvrage dans le fait que Deleule y a critiqué de manière très perspicace la conception selon laquelle le libéralisme n'aurait été qu'une idéologie au service du capitalisme. L'article d'Éléonore LE JALLÉ (Hume: un scepticisme insouciant, pp. 37-46) analyse la section conclusive du livre I du Traité de la nature humaine du philosophe écossais afin de pouvoir expliciter ce que Hume entend par «scepticisme insouciant» (careless scepticism), expression qu'il utilisera dans la Dialogues sur la religion naturelle. Aliénor BERTRAND, dans son Naturalisme et scepticisme: Hume vs Condillac, montre que la méthode qu'adopte le philosophe francais le conduit à interroger les sciences à partir 
de la genèse des langues et des systèmes, plutôt qu'à douter du principe de la causalité. Cette analyse génétique hiérarchise les savoirs de telle sorte que les règles morales échappant à la relativité des coutumes et s'ancrant dans l'universalité du langage naturel, assurent une fondation propre aux sciences de l'homme. Vanessa NUROCK (Naturalisations et scepticisme: pour une naturalisation modérée de la morale, pp.61-73) aborde la question des relations entre psychologie et philosophie. On connait la thèse du remplacement prônée par Quine qui propose de substituer tout simplement la psychologie à l'épistémologie. L'auteure de l'article, avançant sur les pas de Hilary Kornblith préfère à cette version forte de la naturalisation une version faible ou modérée qui permettrait une coexistence et une collaboration des deux disciplines. L'étude de Claude GAUTIER (L'expérience de la discontinuité chez P. Bourdieu: la force des représentations dans les paroles d'autorité, pp.73-89) étudie la critique que Bourdieu adresse à Austin: il s'agit d'identifier comment la sociologie est déterminée, comme point de vue critique sur le monde, à rendre compte de la discontinuité individusociété. Laurent JAFrRO, dans sa Rétorsion du sens commun et la possibilité du scepticisme. Contre Reid (pp. 93-116) reprend l'argument du sens commun auquel Thomas Reid eut recours, contre Hume, afin de prouver l'impossibilité du scepticisme. Ce faisant, Jaffro identifie une tension très forte à l'intérieur de la philosophie de Reid: si sa position peut être qualifiée d'intuitionnisme, sa réfutation du scepticisme l'a conduit à adopter une argumentation pragmatique dont l'esprit paraît bien différent. L'étude de Guillaume LE BLANC (Le libéralisme est-il un individualisme?, pp.118-131) reprend l'analyse par L. Dumont de la naissance de l'individualisme afin de faire apparaître l'envers de l'individu libéral: l'individu dépendant, pris dans une dépendance sociologique et une dépendance relationnelle. Fabienne BRUGÈRE, quant à elle, se demande Le féminisme est-il un libéralisme? (pp. 133-147) et présente les lignes principales d'une critique féministe du libéralisme promouvant une idéologie individualiste et prônant un idéal d'égalité trop abstrait. Sa conclusion est que le libéralisme doit se renouveller avec les critiques féministes qui lui sont adressées et surtout prendre en compte la dignité de la dépendance. L'étude de Frank BURBAGE, intitulé Croissance et développement (pp.149-162) consiste dans une analyse lucide des grands récits de conquête et de croissance caractérisant le capitalisme. Le texte s'efforce de fournir un concept non-dogmatique d'une critique contemporaine de l'économisme. L'article d'Hélène L'HeUillet ( Le sceptique et le cynique ou comment sortir du nihilisme, pp.163-177) étudie les manifestations anciennes et modernes du scepticisme et du cynisme afin de montrer, avec Nietzsche, Dostoïevski et Alan Bloom leur rapport très compliqué avec le nihilisme. Un retour à la philosophie prudente de Hume permettra de retenir les éléments les plus précieux de ces traditions. Le texte de Xavier PAPAIS est une méditation philosophico-poétique sur le sujet des Énigmes (pp.179-191). L'étude de Michèle COHEN HALIMI, consacrée à Siegfried Cracauer, le dernier des détectives (pp. 193-203) traite le geste de S.C. de ne pas publier son livre intitulé Le roman policier. L'auteur se propose d'expliquer cette décision comme un geste politique où la skepsis de l'enquête policière s'élève au genre du traité philosophique pour finir par déserter les formes attendues de la lucidité. David LAPOUJADE (Scepticisme et littérature: le cas Henry James, pp. 205-214) affirme que dans L'image dans le tapis de l'écrivain le scepticisme ne consiste pas dans l'incrédulité finale du critique littéraire (qui cherche le sens d'une œuvre), se confondant avec une certaine amertume et relativisme, mais dans la dissipation de l'objet et son effacement progressif derrière les interprétations qu'il fait naître. L'étude de Michael BIzIou (Kant et Smith critiques du scepticisme de Hume. Les 
avatars du théisme, pp.215-228) montre qu'à propos du scepticisme, les deux philosophes partent d'une critique du concept humien de généralité et ils aboutissent tous les deux à une réhabilitation du théisme. Comme chacun le sait, Kant, dans la Critique de la raison pure, fait du scepticisme un dogmatisme que la pensée critique pourra dépasser. L'étude de Max MARcuzzI (Scepticisme et philosophie transcendentale, pp. 229-241) pense pouvoir montrer que le dispositif des arguments que Kant met en place peut aussi bien déboucher sur des conséquences sceptiques que sur les conclusions critiques. Dans son étude (Du doute existentiel à l'inquiétude politique, figures du scepticisme chez Rousseau, pp. 243-265), Géraldine LEPAN propose une analyse de nombreux thèmes sceptiques présents dans l'œuvre multiforme de Rousseau. L'auteur teste l'idée selon laquelle le scepticisme de R. serait modéré, au sens qu'il accorderait l'existence du monde, d'autrui et de soi et la validité de la raison dans les limites qui lui sont fixées. Stephan VAQUERO, dans son Scepticisme et empirisme éthiques à l'âge classique: le jeu de la prudence baroque (pp.267-279), souligne que le scepticisme baroque se manifeste dans une éthique qu'aucune norme a priori ne peut déterminer: la variabilité et le jeu en sont les éléments incontournables. Il consiste donc moins en une suspension de jugement que dans la tentative de fonder une éthique rompant avec tout fondement métaphysique. L'article de Pierre zaOUI (Le scepticisme comme amour de l'hétérogène. Les leçons de Didier Deleule), apres avoir célébré le séminaire de recherche de D.D. à Paris-X-Nanterre, réfléchit sur les points communs et les écarts qui caractérisent la pensée des philosophes que nous avons l'habitude de qualifier se sceptiques. Enfin, dans un article ("Interpréter au centre", p. 301-310) qui constitue la postface du volume, Denis KAMBOUCHNER analyse les réponses qu'on peut donner à la question qui porte sur les rapports entre l'histoire de la philosophie et la philosophie tout court. 